\title{
Enduring effect of childhood maltreatment on cortisol and heart rate responses to stress: The moderating role of severity of experiences
}

\author{
ISABELLE OUELLET-MORIN,${ }^{a, b, c}$ MARIE-PIER ROBITAILLE, ${ }^{a}$ STÉPHANIE LANGEVIN, ${ }^{a}$ \\ CHRISTINA CANTAVE, ${ }^{a}$ MARA BRENDGEN, ${ }^{c, d}$ AND SONIA J. LUPIEN ${ }^{a, b}$ \\ ${ }^{a}$ University of Montreal; ${ }^{b}$ Research Center of the Montreal Mental Health University Institute; ${ }^{c}$ University of Quebec at Montreal; \\ and ${ }^{d}$ Sainte-Justine Hospital Research Center
}

\begin{abstract}
There is a relative consensus about the detrimental impact of childhood maltreatment on later mental health problems and behavioral difficulties. Prior research suggests that neurophysiological stress mechanisms may partly mediate this association. However, inconsistent findings regarding hypothalamic-pituitaryadrenal axis and sympathetic responses to stress complicate this investigation. Furthermore, the concordance in these two stress systems is not well understood. We tested whether the severity of maltreatment affected the association between maltreatment and cortisol and heart rate (HR) stress responses and the symmetry of these responses. Participants were 155 males (56 maltreated and 99 controls) aged 18 to 35 years. Cortisol and HR were measured in response to the Trier Social Stress Test. Childhood maltreatment, sociodemographic factors, and health-related factors were measured using self-reported questionnaires. Maltreated participants had higher cortisol responses to stress in comparison to controls. However, a shift from moderate to lower to higher cortisol responses was noted as the severity of the experiences increased. Participants exposed to more experiences of maltreatment also showed a greater symmetry between cortisol and HR stress responses. Our findings provide further support for persistent dysregulation of the HPA axis following childhood maltreatment, of which the expression and symmetry with the sympathetic system may change according to the severity of experiences.
\end{abstract}

A relative consensus exists regarding the consequences of childhood maltreatment on emotional, behavioral, and social functioning during childhood and adolescence (Ferrara et al., 2016; Hunt, Slack, \& Berger, 2016). Longitudinal studies have also shown that the negative impact of maltreatment may persist into adulthood and affect multiple domains of life, such as physical and mental health, intimate relationships, employment, and criminal offending (Herrenkohl, Hong, Klika, Herrenkohl, \& Russo, 2013; Mersky \& Topitzes, 2010; Smith, Ireland, \& Thornberry, 2005). Embracing a developmental psychopathology framework could help to understand the negative (and potentially positive) adaptations unfolding over time (Toth \& Cicchetti, 2013). While longitudinal study designs for which measures of functioning and

We are grateful to the participants who have given their time to take part in this study. The data presented in this manuscript have been funded by the HF Guggenheim Foundation. Isabelle Ouellet-Morin is Canada Research Chair in the Developmental Origins of Vulnerability and Resilience, and Stéphanie Langevin is supported by the Fonds de recherche du Québec-Société et Culture. Mara Brendgen was supported by the Fonds de Recherche du Québec-Santé, and Sonia J. Lupien is a Canada Research Chair in Human Stress.

Address correspondence and reprint requests to: Isabelle Ouellet-Morin, School of Criminology, University of Montreal, Research Center of the Montreal Mental Health University Institute and the Research Group on Child Maladjustment, C.P. 6128, succursale Centre-ville, Montréal QC, H3C 3J7, Canada; E-mail: isabelle.ouellet-morin@umontreal.ca. hypothesized causal factors have been collected prospectively remain ideally positioned to make a contribution to the field, additional objectives are pursued. Among them, the identification of the pathways by which maltreatment jeopardizes health and behavioral functioning, through the adoption of multiple levels of analysis and a transactional approach for which the biological, emotional, and psychological processes transcend divisions between normality and abnormality (Beauchaine \& McNulty, 2013; Cicchetti, 2016). To date, though, the mechanisms underlying the long-lasting impact of maltreatment on functioning remain elusive, and are often examined in isolation and according to artificially truncated sources of influences.

The hypothalamic-pituitary-adrenal (HPA) axis and the sympathetic nervous system (SNS) are hypothesized to play a central role in the association between early adversity and health (Doom \& Gunnar, 2013; Lupien, McEwen, Gunnar, \& Heim, 2009). While a flattened pattern of diurnal cortisol secretion has been reported in maltreated children with some consistency (Bernard, Frost, Bennett, \& Lindhiem, 2017; Cicchetti, Rogosch, Gunnar, \& Toth, 2010; Power, Thomas, Li, \& Hertzman, 2012), both lower (Bernard et al., 2017; Bruce, Fisher, Pears, \& Levine, 2009; Power et al., 2012) and higher (Bruce et al., 2009; Bugental, Martorell, \& Barraza, 2003; Engert, Efanov, Dedovic, Dagher, \& Pruessner, 2011; Fries, Shirtcliff, \& Pollak, 2008; Saridjan 
et al., 2010) basal cortisol levels are noted. Inconsistent findings have also been reported regarding the directionality of the association between maltreatment and cortisol responses to psychosocial stress. Adolescents and adults who had been maltreated as children showed either higher (Harkness, Stewart, \& Wynne-Edwards, 2011; Heim et al., 2000; Sullivan, Bennett, \& Lewis, 2013), no difference (Hagan, Roubinov, Mistler, \& Luecken, 2014; Suzuki, Poon, Papadopoulos, Kumari, \& Cleare, 2014) or lower cortisol responses to stress than controls (Carpenter et al., 2007; Carpenter, Shattuck, Tyrka, Geracioti, \& Price, 2011; Cook, Chaplin, Sinha, Tebes, \& Mayes, 2012; Elzinga et al., 2008; Lovallo, Farag, Sorocco, Cohoon, \& Vincent, 2012; MacMillan et al., 2009; OuelletMorin et al., 2011; Peckins, Dockray, Eckenrode, Heaton, \& Susman, 2012; Trickett, Gordis, Peckins, \& Susman, 2014; Voellmin et al., 2015). Mixed findings also exist in regard to the SNS response to stress (Cook et al., 2012; De Bellis, Lefter, Trickett, \& Putnam, 1994; Gordis, Feres, Olezeski, Rabkin, \& Trickett, 2010; MacMillan et al., 2009; Voellmin et al., 2015).

A common source of inconsistency among studies examining the association between maltreatment and these stress response systems is the selected methodology. Besides the confounding effects of factors, such as the time of day, medications, and current symptomatology, another, more concealed source of discrepancy may lie in the measure of maltreatment. Most studies relied on data collected in participants drawn from the general population, including individuals who were maltreated (with or without official records) and others who were not. Without the necessary precautions (e.g., overrepresentation of participants who report maltreatment), this strategy may reduce the heterogeneity of the maltreatment experiences captured in these samples, in terms of frequency and severity. Moreover, the use of a dichotomous index of maltreatment (presence vs. absence) may be problematic if the association with the stress response systems changes direction (i.e., is not linear) as maltreatment becomes more severe. The presence of a point of inflexion, a shift, in cortisol response to stress along the continuum of maltreatment severity echoes in part the stress inoculation model, which proposes that moderate stress dampens cortisol response to future stress (Parker, Buckmaster, Schatzberg, \& Lyons, 2004). However, exposure to more severe stress may not be "inoculating" per se, but could sensitize the HPA axis to subsequent stress (Obradovic, 2012). Consequently, the restricted range of maltreatment captured in some studies, because of the participants' selection procedures or due to how maltreatment was measured or analyzed, may not adequately depict the association between maltreatment and the stress response systems. Further tests are warranted.

The impact of these methodological concerns depends, among other things, on whether or not the association linking maltreatment and the stress response systems is expected to be linear. If maltreatment is linearly associated with cortisol (i.e., lower or higher cortisol response as maltreatment severity increases), the constrained range of maltreatment experiences or the use of a dichotomous index bear no (or fewer) conse- quences in understanding this association. In contrast, if a shift in the physiological stress response exists somewhere along the maltreatment distribution, only a partial representation of the maltreatment-cortisol association would be revealed. Such a possibility cannot be easily assessed from existing studies. For example, higher levels of adverse childhood experiences (ACEs) were associated with lower cortisol responses to stress in a sample of young women (Voellmin et al., 2015). However, it is not clear whether, in a sample of participants who reported an average of 2.8 ACEs, those who reported the highest level of adversity (i.e., 4 or more ACEs) were confronted by moderate or higher levels of adversity so that lower or higher patterns of cortisol response could be present along the ACE distribution. As both lower and higher cortisol responses to stress have been reported following maltreatment, a closer look at the directionality of this association, according to the severity of maltreatment experiences, is warranted.

Another source of inconsistency may be that studies generally examine one stress response system at a time, precluding a more systematic, integrated view of how maltreatment may have long-lasting effects on these systems (Bauer, Quas, \& Boyce, 2002). The locus coeruleus-norepinephrine/SNS and the HPA axis do not always present symmetrical (lagged) responses to stress (Allwood, Handwerger, Kivlighan, Granger, \& Stroud, 2011; Bae et al., 2015; Cook et al., 2012; El-Sheikh, Erath, Buckhalt, Granger, \& Mize, 2008; Gordis, Granger, Susman, \& Trickett, 2006; van Goozen et al., 1998). Moreover, despite their neural connections, these stress systems have distinct secretion time course, effects on targeted tissues, and regulatory mechanisms (Sapolsky, Romero, \& Munck, 2000). Important individual variability in the HPA axis and SNS responses to stress have been reported (Kudielka, Hellhammer, \& Wust, 2009), so that while some individuals show marked responses in both systems when confronted by stress, only one stress system may be activated in others (Bauer et al., 2002). According to the biological sensitivity to context model (Boyce \& Ellis, 2005), genetically and environmentally mediating factors have the potential to calibrate the stress systems. It is thus possible that the specific genetic and environmental etiology of the HPA axis and SNS may prompt asymmetric responses to stress. On a related point, the biological sensitivity to context model underlines how the integrated actions and connections of the stress systems should be understood in their ecological contexts, past or present (Ellis \& Boyce, 2008). However, the possibility that the symmetry of the HPA and SNS responses to stress vary according to childhood maltreatment has rarely been investigated (for an exception, see Gordis, Granger, Susman, \& Trickett, 2008). In summary, the restricted range of maltreatment captured in some studies, the overlook of potential nonlinear association between these constructs as well as the omission of testing the possibility that maltreatment severity affects the symmetry of HPA and SNS responses to stress, may obscure how they jointly affect later vulnerability to psychopathology. 
The present study had three objectives. We examined whether young adults exposed to childhood maltreatment, as defined using the standardized Childhood Trauma Questionnaire (CTQ) guidelines (Bernstein \& Fink, 1998), had a disrupted pattern of stress responses in comparison to those who did not reach that threshold, using cortisol and heart rate (HR) as markers of HPA axis and SNS reactivity to stress. This dichotomous index of maltreatment experiences, however, would not allow uncovering the nonlinear patterns of associations, if present. To this end, we investigated if the cortisol and HR responses to stress varied according to maltreatment severity. Here, we operationalized maltreatment experiences so that individual variation within the CTQbased nonmaltreated participants could be depicted along with their differential patterns of associations with the stress response systems. Finally, we explored whether the symmetry between cortisol and HR responses to stress also varied as a function of maltreatment severity.

\section{Method}

\section{Sample}

Because the study's general objective was to understand the biosocial roots of general aggression, including verbal and physical aggression, and that these behaviors are manifested more frequently by men than by women (Archer, 2004), the sample only comprised men. Moreover, uncertainty remains about the existence of sex differences in cortisol response to stress (Carpenter et al., 2009; Kudielka, BuskeKirschbaum, Hellhammer, \& Kirschbaum, 2004) and regarding the maltreatment-cortisol associations (Carpenter et al., 2009; Negriff, Saxbe, \& Trickett, 2015). Because we could not reach the statistical power required to adequately test these possible sexually dimorphic associations, only men were initially investigated. Participants were recruited using ads posted online and on public billboards inviting them to participate in a study about early life experiences. Trained research assistants conducted a phone interview with interested individuals, screening for health and about experiences of childhood maltreatment using the short form of the CTQ (Bernstein \& Fink, 1998). The sample included 155 participants aged from 18 to 35 years $(M=24.10$, $S D=3.70)$.

\section{Procedure}

We invited the participants to take part in our study, lasting about $3 \mathrm{hr} 30 \mathrm{~min}$. During that time, the participants took part in the Trier Social Stress Test (TSST), a well-established, standardized stress paradigm that induces social-evaluative threat by subjecting participants to a 5-min mock job interview in front of a "panel of behavioral experts," and followed by $5 \mathrm{~min}$ of mental arithmetic. Participants communicated with the panel using an intercom and were filmed with a video camera in a stand-up position in front of a one-way window
(Andrews et al., 2007). Both the "panel-in" and "panel-out" methods have been shown to elicit reliable cortisol responses in laboratory settings (Andrews et al., 2007). The TSST took place in the early afternoon for all participants $(M=13: 41$, $S D=0: 53)$. Ethical approval was granted by the Research Center of the Montreal Mental Health University Institute Ethics Committee (Montréal, Canada).

\section{Measures}

$C T Q-S F$. The CTQ-SF inquiries information about emotional, physical, and sexual abuse and neglect that have occurred before age 18 and have been selected because of its demonstrated validity in community samples (Bernstein et al., 2003). Participants responded to each of the 28 items in the context of "when you were growing up" and answered according to a 5-point Likert scale ranging from $1=$ never true to $5=$ very often true, leading to total scores of 5 to 25 on each subscale (plus 3 items of validity). The CTQSF included items such as "People in my family said hurtful or insulting things to me" (emotional abuse) and "I was punished with a belt, a board, a cord, or some other hard object" (physical abuse). To test the first objective of the study, we identified 56 men (35.9\%) who reported experiences suggesting the occurrence of at least one type of maltreatment (i.e., CTQ-based maltreatment) using the manual's recommended classification scores (Bernstein \& Fink, 1998). The experiences of the remaining participants $(n=99$; controls) did not reach that threshold (i.e., CTQ-based nonmaltreatment). Being identified as positive for a category corresponds with endorsing a number of experiences as "often true." The CTQ has good internal consistency and criterion validity in clinical and community samples and convergent reliability with clinical assessments of abuse is high (Bernstein et al., 2003). Acceptable internal consistency was also found in this sample (Cronbach $\alpha=0.78$ ). To test the second objective of the study, we calculated the total scale of maltreatment by summing up all 25 items, as an index of maltreatment severity (total sample: $M=37.43, S D=11.04$ ), and derived three equal groups of participants who reported increasing levels of maltreatment experiences, thereafter referred to as no (few) maltreatment experiences (CTQ total score $\leq 31$ ); some experiences of maltreatment (CTQ total score 32-40); and more experiences of maltreatment (CTQ total score $\geq$ 41). The groups based on the CTQ's guidelines for maltreatment, $M=30.85, S D=3.96$ and $M=49.18, S D=9.81$, respectively; $F(1,154)=271.15, p<.001$, as well as the groups derived according to the total CTQ score, significantly differed in the levels of maltreatment reported, $M=28.12, S D$ $=2.00, M=34.91, S D=2.63$, and $M=50.78, S D=9.07$, respectively; $F(2,153)=239.02, p<.001$, with post hoc analyses showed significant differences between each group $(p s<.001)$.

Stress biomarkers. Cortisol was measured through the collection of five saliva samples via passive drool. The first two 
samples were collected 2 and $20 \mathrm{~min}$ before the TSST. The third, fourth, and fifth samples were collected 15, 25, and 35 min after the beginning of the TSST. Saliva samples were stored in a $-20{ }^{\circ} \mathrm{C}$ freezer and analyzed in a single batch with a high sensitivity enzyme immune assay kit (Salimetrics State College, PA, Catalogue No. 1-3102). The range of detection for this assay is between 0.012 and $3 \mathrm{ug} / \mathrm{dl}$ and the intra- and interassay coefficients of variation were $4.1 \%$ and $8.3 \%$, respectively. All samples were assayed in duplicates, Winsorized, and log-transformed prior to statistical analyses.

$H R$ was measured using an Omron 7 Series Plus BP765 Automatic Blood Pressure Monitor, which records blood pressures in a noninvasive manner using a cuff placed on the upper arm. HR was measured, at a 2-min interval, three times prior to and six times during the TSST.

\section{Risk factors and covariates}

Information about the sociodemographic, health, and lifestyle factors, including age, occupation, and cigarette, alcohol, and other drugs consumption were enquired during the initial phone interview. We also asked the participants to complete the Beck Depression Inventory during the laboratory visit, which is a 21-question multiple-choice self-report inventory measuring depressive symptoms and severity (Beck, Steer, \& Brown, 1996).

\section{Statistical analyses}

Before conducting the main analyses, we identified, from a wide range of health-related factors (e.g., medication, cigarettes, alcohol and drug use, and allergies), those uniquely associated with cortisol and HR. Two were identified for cortisol: being a smoker and having had a flu in the past month and one for HR: anti-inflammatory medication. These variables were statistically controlled for in all analyses. We tested the study hypotheses in three steps. First, we tested whether the participants showed a distinct pattern of stress response according to the CTQ-based maltreatment status using a repeated-measures analysis of variance (ANOVA; cortisol) and growth curve analyses (HR). A repeated-measures ANOVA was preferred for cortisol because we had no missing data and the saliva samples were all collected at predetermined, constant times in relation to the beginning of the TSST. Greenhouse-Geisser corrections for repeated measures were reported to correct for the violated sphericity assumption. Because missing data occurred for HR due to technical difficulties, we conducted growth curve analyses using full information maximum likelihood estimation in MPlus (Version 6.11; Muthén \& Muthén, 1998-2011). Second, we wanted to expand on the previous analysis and test if distinct, nonlinear patterns of cortisol and HR responses to stress could be detected according to the severity of maltreatment. As described in the Method, we split the total sample in three groups according to the continuously distributed total CTQ score of maltreatment, yielding to participants who reported no (few) maltreatment experiences; some experiences of maltreatment; or more experiences of maltreatment. Tertiles were chosen to allow a similar number of participants in each group and sufficient statistical power to test the study's hypotheses. Third, we explored whether the symmetry between cortisol and HR responses to stress was moderated by maltreatment severity using the regression model included in the SPSS's macro PROCESS (Hayes, 2013).

\section{Results}

Did the TSST elicit a significant cortisol stress response, and could distinct patterns of cortisol responses to stress be detected according to the CTQ-based maltreatment status?

A repeated measures ANOVA showed that the TSST elicited a robust and significant increase in cortisol, time: $F(2.05$, $311.56)=57.99, p<.001$. Figure 1 illustrates the patterns of secretion for the participants for whom the experiences reached the CTQ's threshold for maltreatment (solid line) and those for whom it did not (dashed line). The CTQ-based maltreated participants had higher cortisol responses to the TSST, Time $\times$ CTQ-Based Maltreatment: $F(2.08,313.27)$ $=2.89, p=.05$, and a trend for significantly higher cortisol levels throughout the TSST, CTQ-based maltreatment: $F(1,151)=3.69, p=.06$, in comparison to the CTQ-based nonmaltreatment group. Specifically, while the two groups did not differ prior to the TSST, $-20 \mathrm{~min}: F(1,154)=$ $1.01, p=.32$; -2 min: $F(1,154)=0.88, p=.35$, distinct responses emerged subsequently, $F(1,151)=4.60, p=.03$.

\section{Did distinct patterns of cortisol responses to stress emerge according to maltreatment severity?}

Distinct cortisol responses were noted according to maltreatment severity, $F(4.17,312.89)=2.84, p=.02$. Figure 2 shows that while the three groups had similar initial cortisol levels, participants who reported more experiences of maltreatment had higher cortisol responses to the TSST in comparison to the remaining groups. We formally tested this observation by contrasting the groups two by two. Participants who reported more experiences of maltreatment had higher cortisol responses than those who reported some experiences, $F(2.00,186.16)=5.18, p=.006$, but did not differ significantly from the participants with no or few experiences, $F(2.10,217.95)=1.45, p=.24$. Moreover, participants who reported some experiences of maltreatment had lower responses than controls, $F(1,101)=3.89, p=.05$. Altogether, these findings suggest lower responses when maltreatment departs from the no (or few) experiences, followed by higher cortisol levels in participants confronted to more experiences of maltreatment, a finding hereafter referred to as a shift from moderate to lower to higher responses as maltreatment severity increased. 


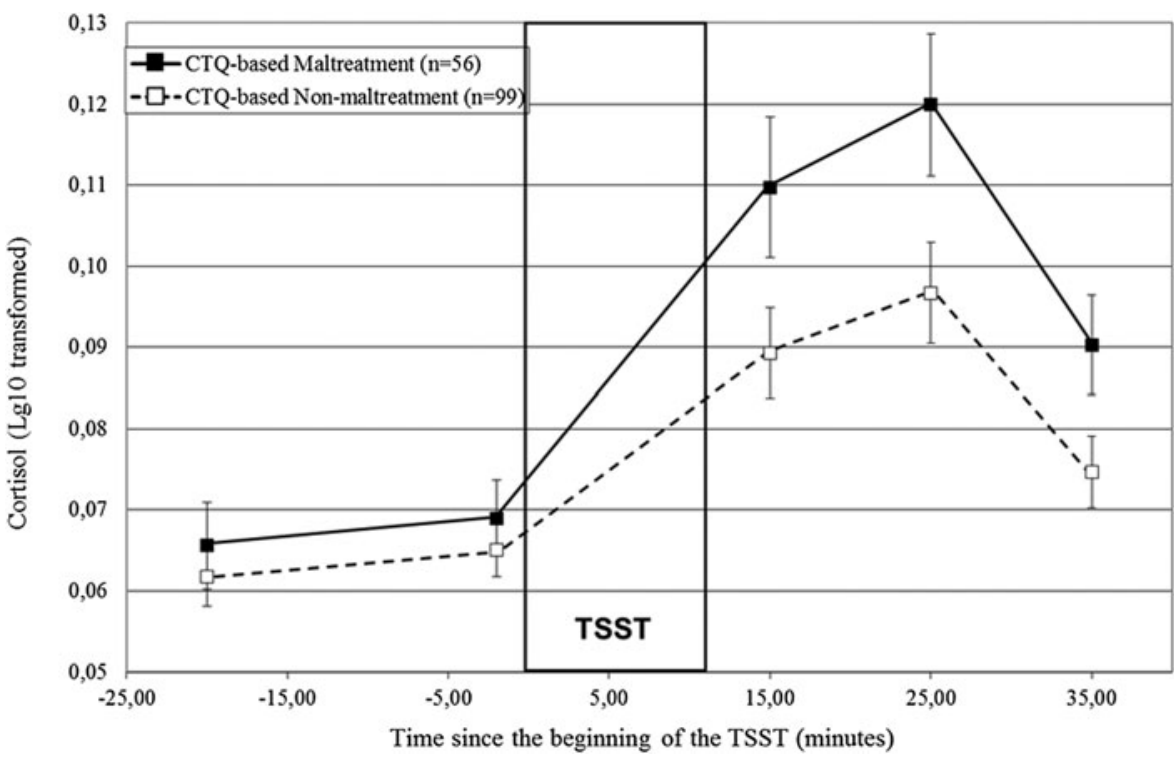

Figure 1. Cortisol responses ( $\pm S E M$ ) to the TSST according to CTQ-based maltreatment status. SEM, standard mean error; $n$, number of participants; TSST, Trier Social Stress Test; CTQ, Childhood Trauma Questionnaire.

Were similar patterns of findings observed for $H R$ response to stress?

As reported in the online-only Supplementary Table S.1, participants showed, on average, a significant HR increase during the TSST, $B=6.95(0.41)$, critical ratio $=16.96, p<.001$, which did not vary according to the CTQ-based maltreatment groups, $B=-0.31(0.85)$, critical ratio $=-0.36, p=.72$ (Figure 3). In addition, no differences in HR could be detected according to maltreatment severity, main level: $B=-0.31$ (1.01), critical ratio $=-0.31, p=.76$; stress response: $B=$ $-0.61(0.48)$, critical ratio $=-1.26, p=.21$ (Figure 4).

\section{Other potential confounders}

In addition to the factors affecting cortisol and HR, we explored whether other factors differed between participants

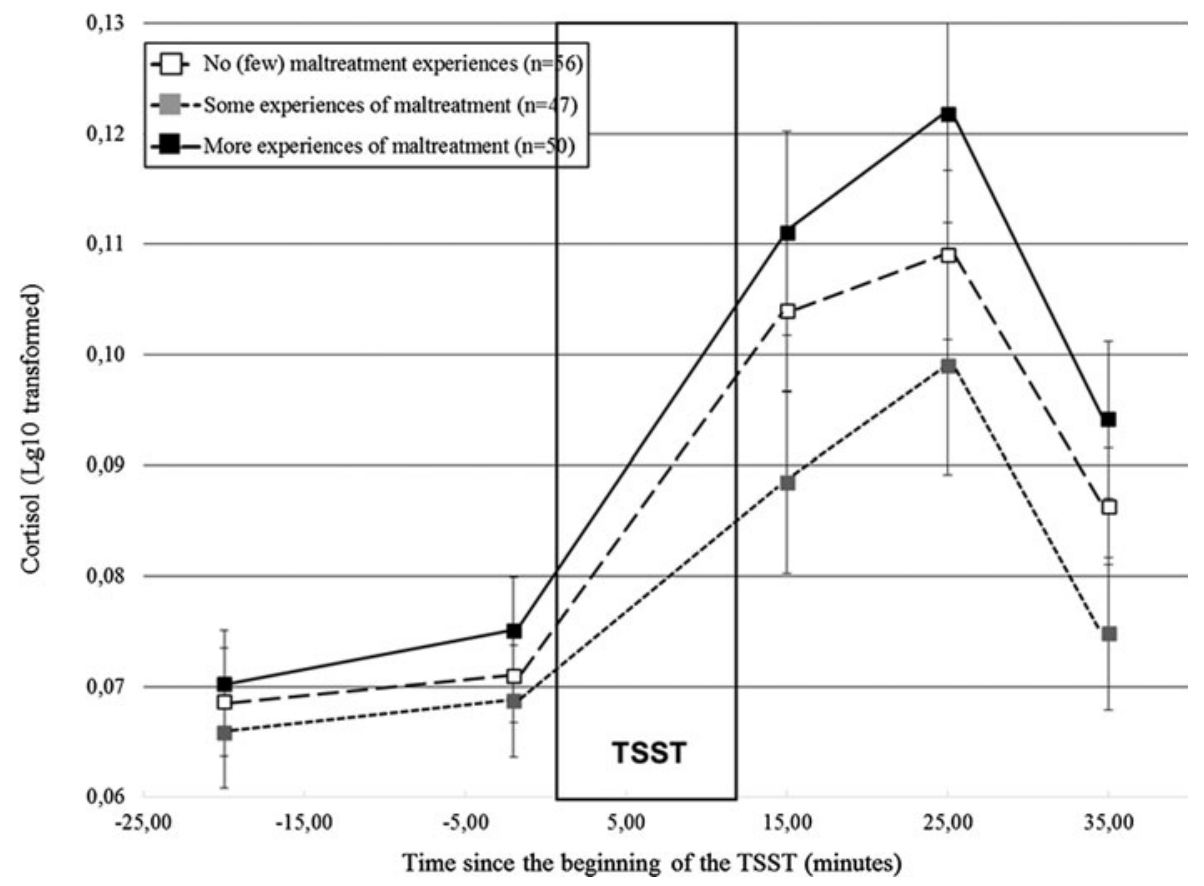

Figure 2. Cortisol responses ( $\pm S E M$ ) to the TSST according to the severity of maltreatment. SEM, standard mean error; $n$, number of participants; TSST, Trier Social Stress Test. 


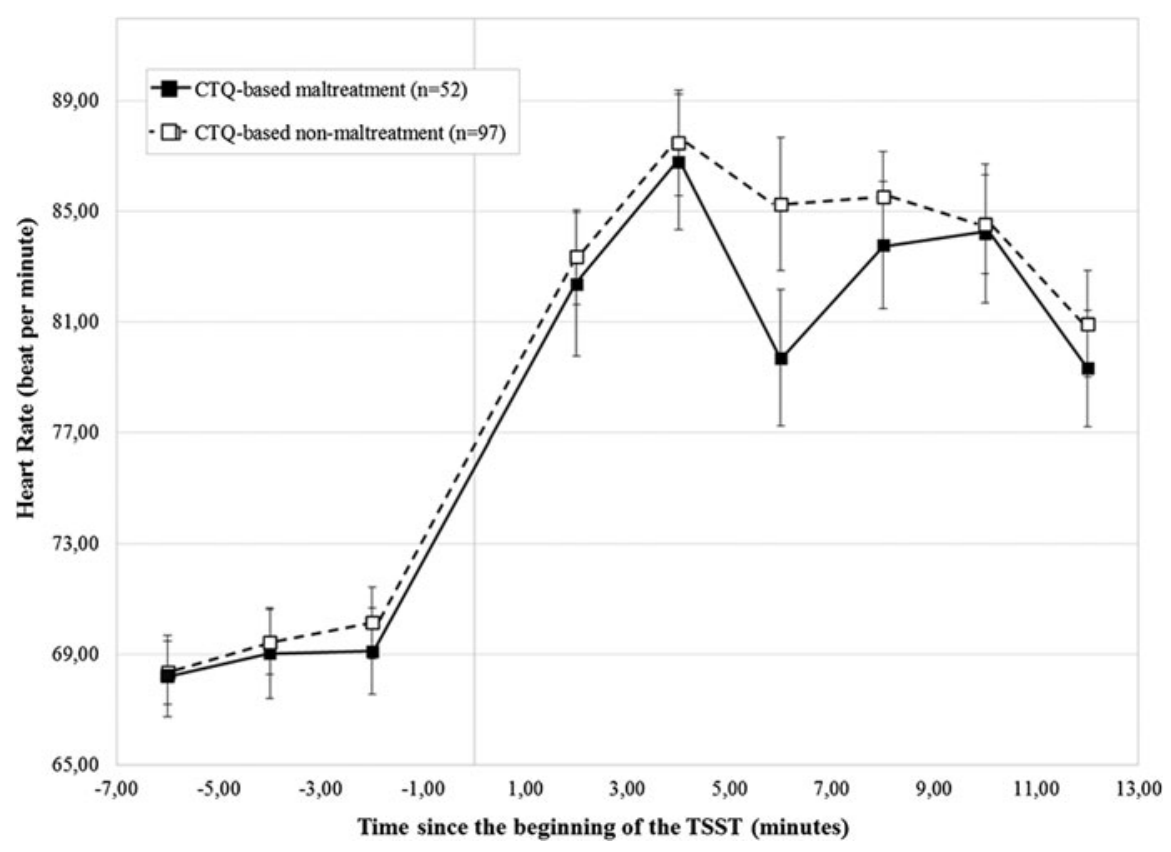

Figure 3. Heart rate responses ( $\pm S E M$ ) to the TSST according to CTQ-based maltreatment status. SEM, standard mean error; $n$, number of participants; TSST, Trier Social Stress Test; CTQ, Childhood Trauma Questionnaire.

who reported CTQ-based maltreatment and those who did not (Table 1). Participants assigned to the CTQ-based maltreatment group have had more flu in the previous month and more depressive symptoms than those who did not. While the first factor was already accounted for in the previous analyses, we reran all the analyses to ensure that the depressive symptomatology did not explain away the findings. Participants who reported maltreatment that reached the CTQ-based threshold still showed higher cortisol responses to the TSST in comparison to those who did not, $F(1.86,276.14)=$ $4.07, p=.02$. The distinct patterns of cortisol responses to stress also remained according to increasing levels of maltreatment severity, $F(3.75,275.94)=3.71, p=.007$, for which lower responses were noted for participants who reported some experiences of maltreatment in comparison to those who reported more experiences, $F(1.80,162.24)=$ $6.32, p=.003$. Findings also remained unchanged for HR.

\section{Did the symmetry between cortisol and HR vary according to maltreatment severity?}

Maltreatment severity moderated the association between cortisol and HR responses to stress, $F(1,146)=5.30, p=$ .02 . To explore the direction and relative magnitude of these associations according to maltreatment severity, we conduct post hoc Pearson correlations. As illustrated in Figure 5, no significant associations were observed for participants exposed to no (or few; $r=-.17, p=.22$ ) or some experiences of maltreatment $(r=.20, p=.18$ ), whereas a significant association emerged for those who reported more experiences of maltreatment $(r=.34, p=.02)$.

\section{Discussion}

The present study examined the association between childhood maltreatment, cortisol, and HR responses to a psychosocial stress in early adulthood. Consistent with previous findings, we found indications of both lower and higher cortisol responses to stress in the context of maltreatment. As previously hypothesized (Del Giudice, Ellis, \& Shirtcliff, 2011; Obradovic, 2012), these patterns of secretion may embody different expressions of changes made by the HPA axis in an attempt to adapt to the perceived environmental demands according to past experiences, such as childhood maltreatment.

Three features of these findings are noteworthy. First, the lower cortisol response noted in participants who reported only some experiences of maltreatment falls in line with the findings from studies that have recruited participants from the general population (Carpenter et al., 2007, 2011; Elzinga et al., 2008; Lovallo et al., 2012; Ouellet-Morin et al., 2011; Peckins et al., 2012; Peckins, Susman, Negriff, Noll, \& Trickett, 2015) and Child Protective Services (Trickett et al., 2014). A direct comparison of the relative severity of maltreatment captured in these studies is difficult to make, however. More promising is the consideration that the association between maltreatment and cortisol reactivity may not be linear. Many biological and psychological processes are expected to be nonlinear because of the regulating actions of several intertwined structures and systems. Still, linear models remain the analytical strategy of choice (Mattei, 2014; Young \& Benton, 2015). HPA axis reactivity to stress is similarly modulated by several brain structures processing the cognitive and emotional information according to present 


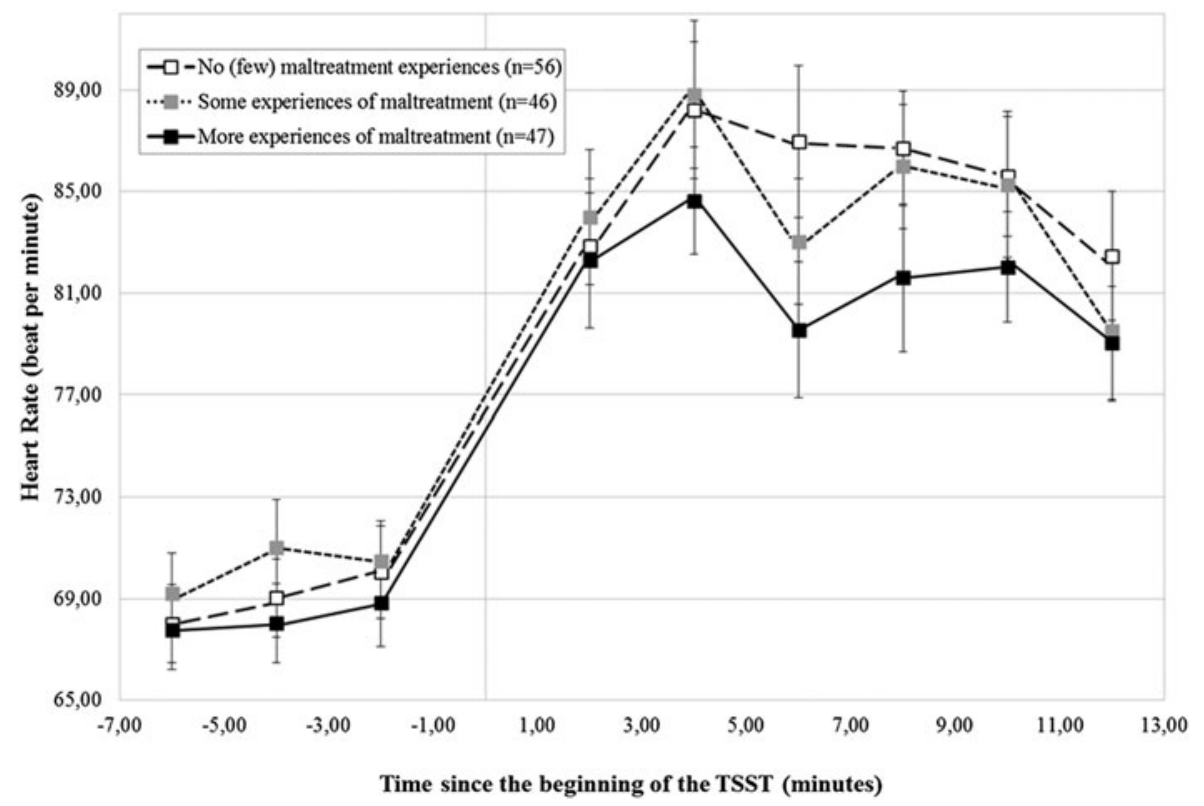

Figure 4. Heart rate ( $\pm S E M$ ) to the TSST according to the severity of maltreatment. SEM, standard mean error; $n$, number of participants; TSST, Trier Social Stress Test.

and past contexts (e.g., frontal cortex, the hippocampus, and the amygdala), which jointly regulate cortisol secretion through excitatory and negative-feedback pathways at the level of the hypothalamus, the pituitary, and the adrenal glands (Gunnar \& Vazquez, 2006). Multiple embedded systems also interact at a molecular level to regulate the initiation, amplitude, and termination of the stress response, including genetic and epigenetic processes, glucocorticoid and mineralocorticoid receptors, endogenous sex steroids, and oral contraceptives (de Kloet, 2014; Hamstra, de Kloet, van Hemert, de Rijk, \& Van der Does, 2015; Houtepen et al., 2016; Kudielka et al., 2009; Meaney, 2010; OuelletMorin et al., 2013). The shift from moderate to lower to higher cortisol responses as maltreatment severity increased suggests we should revisit the assumption of linearity when investigating the association between maltreatment and HPA axis reactivity. Nonlinear models may help to depict more precisely the changes (e.g., directionality and strength) of the association between maltreatment and cortisol response to stress according to the severity of the experiences, to better document individual variation in this change and the determinants affecting it (e.g., relative severity and chronicity of exposure, timing; for an in-depth discussion on how nonlinear growth models may be helpful in developmental research, see Grimm, Ram, \& Hamagami, 2011).

Second, the lower cortisol responses in participants who reported some experiences of maltreatment are also consistent with the stress inoculation model, according to which dam-

Table 1. Participant characteristics in the total sample and according reported experiences of maltreatment

\begin{tabular}{|c|c|c|c|c|c|}
\hline $\begin{array}{c}\text { Potential } \\
\text { confounders }\end{array}$ & $\begin{array}{c}\text { Total sample } \\
\%(n) \text { or mean }(S D) \\
N=155\end{array}$ & $\begin{array}{c}\text { CTQ-based } \\
\text { nonmaltreated } \\
\%(n) \text { or mean }(S D) \\
n=99\end{array}$ & $\begin{array}{c}\text { CTQ-based } \\
\text { maltreated } \\
\%(n) \text { or mean }(S D) \\
n=56\end{array}$ & $x^{2}$ & $t$ \\
\hline Age & $24.1(S D=3.7)$ & $24.10(S D=3.7)$ & $24.0(S D=3.6)$ & - & 0.08 \\
\hline Student (vs. other) & $64.7 \%(n=101)$ & $62.0 \%(n=62)$ & $69.6 \%(n=39)$ & 0.91 & - \\
\hline Single (yes/no) & $85.9 \%(n=134)$ & $85.0 \%(n=85)$ & $87.5 \%(n=49)$ & 0.18 & - \\
\hline Weight $(\mathrm{kg})$ & $76.4(S D=14.8)$ & $76.1(S D=14.6)$ & $76.9(S D=15.4)$ & - & -0.32 \\
\hline Number of alcohol consumption per week & $3.4(S D=3.9)$ & $3.2(S D=3.8)$ & $3.7(S D=4.0)$ & - & -0.73 \\
\hline Smoking (yes/no) & $17.9 \%(n=28)$ & $14.0 \%(n=14)$ & $25.0 \%(n=14)$ & 2.94 & - \\
\hline Drug consumption (yes/no) & $25.0 \%(n=39)$ & $23.0 \%(n=23)$ & $28.6 \%(n=16)$ & 0.59 & - \\
\hline Had a flu in the last month (yes/no) & $23.1 \%(n=36)$ & $17.0 \%(n=17)$ & $33.9 \%(n=19)$ & $5.79 *$ & - \\
\hline Depressive symptomatology & $10.48(S D=8.79)$ & $8.82(S D=7.30)$ & $13.45(S D=10.39)$ & - & $-2.95 * *$ \\
\hline
\end{tabular}

Note: SD, standard deviation; $n$, number of participants; kg, kilogram. ${ }^{*} p<.05 ; * * p<.01 ; * * *<.001$. 

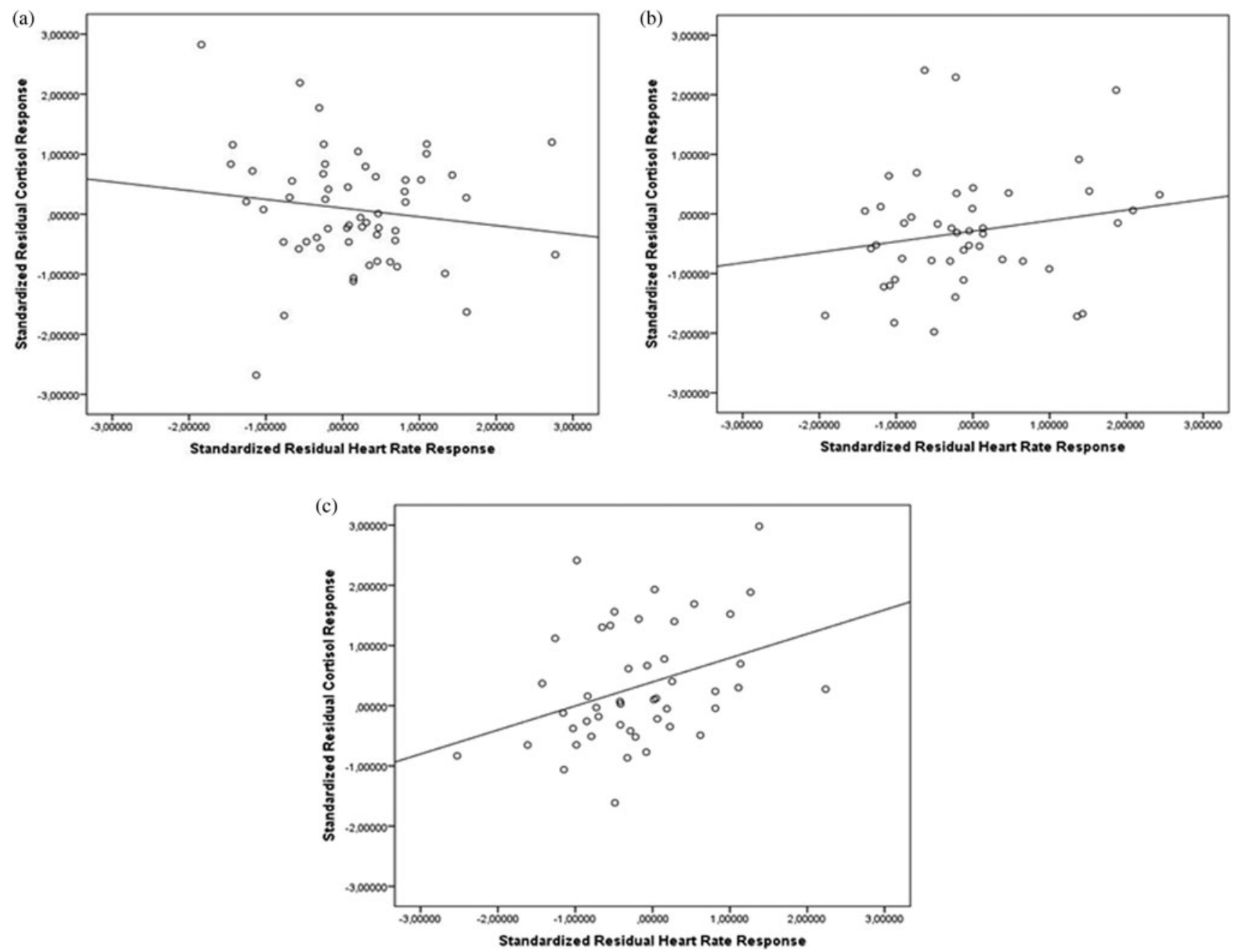

Figure 5. Associations between cortisol and heart rate responses to the TSST according to the severity of maltreatment. (a) No (few) maltreatment experiences, (b) some experiences of maltreatment, and (c) more experiences of maltreatment. TSST, Trier Social Stress Test.

pened activations of the HPA axis befall milder to moderate exposure to stress (Crofton, Zhang, \& Green, 2015; Parker et al., 2004). Nevertheless, while lower cortisol response to stress may protect the body against excessive exposure over time, it may not be synonymous to resilience, which refers to better than anticipated adaptation to detrimental environmental influences (Luthar \& Zelazo, 2003; Shirtcliff, Peres, Dismukes, Lee, \& Phan, 2014). Caution may also be warranted given that lower basal cortisol levels and response to stress have been associated with antisocial behaviors and posttraumatic stress disorder (Boks et al., 2016; Lupien et al., 2006; Susman, 2006; van Goozen, Fairchild, Snoek, \& Harold, 2007). Future studies need to identify the mechanisms by which maltreatment confers vulnerability (or resilience) to mental health or behavioral problems (Bowes \& Jaffee, 2013).

Third, the shift from moderate to lower to higher cortisol responses to the TSST as maltreatment increased in severity may be better understood, in all its complexity, as diverse ex- pressions of the HPA axis attempting to adapt to distinct maltreatment experiences. This is consistent with the allostatic load (Juster, McEwen, \& Lupien, 2010; McEwen, 1998) and the adaptive calibration models (Del Giudice et al., 2011), proposing that the HPA axis' responses reflect longterm individual trajectories that can be recalibrated according to environmental circumstances and for which adaptive and detrimental costs may ensue. Our findings also parallel Del Giudice et al.'s (2012) model predicting a succession of moderate, lower, and higher stress responses in safe, moderately stressful and risky environments. One difference lies at the very end of the continuum where severe and traumatic environments are hypothesized to result in even lower (males) and higher (females) responses (Del Giudice et al., 2011). However, others have not found evidence for that fourth cortisol profile either (Peckins et al., 2015).

More generally, other studies have also reported a shift for basal cortisol activity, where higher morning levels were noted in moderate adversity while lower levels were detected 
in more severe conditions (Gustafsson, Anckarsater, Lichtenstein, Nelson, \& Gustafsson, 2010; van der Vegt, van der Ende, Kirschbaum, Verhulst, \& Tiemeier, 2009). Higher to lower morning basal cortisol levels were also observed in abused females, from adolescence to adulthood (Trickett, Noll, Susman, Shenk, \& Putnam, 2010; see also Trickett, Noll, \& Putnam, 2011)). Likewise, Doom, Cicchetti, and Rogosch (2014) reported higher to lower basal cortisol levels over time in maltreated school-aged children. This findings may also depend, in addition to maltreatment severity, on factors such as the age, duration and type of maltreatment, as well as time passed since the end of maltreatment, and the presence of co-occurring factors enhancing vulnerability (e.g., puberty) or promoting resilience (e.g., social support; Gunnar \& Vazquez, 2006; Lupien et al., 2009; Miller, Chen, \& Zhou, 2007). Future studies should also investigate further whether maltreated individuals with lower or higher patterns of response to stress are more vulnerable to mental health problems and behavioral difficulties. Drawing from the adaptive calibration model, the recalibration of the stress systems may be adaptive by amplifying, or filtering the information present in the environment, in order to avoid further aggression or to develop a sense of normality, for instance. Tradeoffs may, however, be present, such as increased sensitivity to social judgment, taxing even further the individuals' already solicited stress systems and coping mechanisms. The higher risks of internalizing (e.g., depression) and externalizing (e.g., antisocial behavior) problems in individuals with higher and lower cortisol activity, respectively, suggest the possibility that distinct costs may arise depending of the recalibration of the stress systems (Struber, Struber, \& Roth, 2014; Susman, 2006). Longitudinal studies investigating the recalibration of the stress response systems as they unfold and according to the numerous intervening factors would be uniquely suited to depict the time-varying tradeoffs following these adaptations (Mead, Beauchaine, \& Shannon, 2010).

Nevertheless, it is only among participants who reported more experiences of maltreatment that a significant degree of symmetry emerged between cortisol and HR responses to stress. This finding, albeit preliminary and exploratory in nature, echoes in part the permissive or preparative actions of glucocorticoids on the SNS, previously suggested by Sapolsky, Romero, and Munck (2000). Both the permissive and the preparative actions of glucocorticoids theoretically enhance the body's arousal to a new or repeated appearance of a stressor. In this cross-sectional study for which no measure of diurnal secretion was made, we can only speculate about the possibility that the exposure to higher levels of maltreatment could have also induced persistent changes in basal secretion, so that an enhancing action of glucocorticoids on HR and cortisol responses to the TSST may have been taken place (i.e., permissive actions). Alternatively, the higher cortisol responses to stress observed in participants who reported more maltreatment experiences may reflect a stable pattern of responsivity (i.e., preparative actions). The time-varying and reciprocal nature of these influences complicates the understanding of the symmetry between cortisol and HR responses to stress and its adaptive value (or cost) on health and functioning. Future studies should measure both basal and reactive HPA axis and SNS to disentangle the potential permissive and/or preparative effects of glucocorticoids on the stress systems in increasing levels of maltreatment.

The present findings should be considered in light of some limitations. First, childhood maltreatment was assessed using the CTQ-SF, a well-validated and widely used questionnaire, for which the recall of experiences may, however, have been tainted by memory loss and bias. Nevertheless, other findings suggested that recall bias emerging as a result of directed forgetting and relabeling (Epstein \& Bottoms, 2002), for instance, accounted for less than $1 \%$ of the maltreatment's variance and that the use of retrospective reports has little effect on the investigation of its long-term impact on functioning (Fergusson, Horwood, \& Boden, 2011). This strategy may even be preferable to the sole consideration of recent exposure to stress that may not have lasted long enough to induce persisting changes in cortisol response to stress (Peckins et al., 2015). Second, we did not examine the possibility that distinct associations may have emerged as a function of maltreatment subtypes (e.g., emotional abuse and neglect) nor did we take into account the fact that the items included in the CTQ are not equally severe in their impact on children (Barnett, Manly, \& Cicchetti, 1993). On a related point, our measure could not untangle the impact of frequency from the severity of maltreatment, two concepts shown to be differentially associated with behavioral outcomes (Jackson, Gabrielli, Fleming, Tunno, \& Makanui, 2014). Future research with more participants who were confronted with maltreatment and for whom the experiences could be operationalized more homogenously based on the information available in the official records holds the promise of better describing the impact of this complex, multifaceted concept on the stress response systems and functioning (see Mennen, Kim, Sang, $\&$ Trickett, 2010). Third, since our measure of maltreatment encompassed all experiences that have occurred before 18 years of age, we could not test whether the nature or timing further affected cortisol responses in a nonlinear manner. More generally, the absence of prospectively and repeatedly collected measures in this study impeded us from investigating the developmental processes and time-varying individual and environmental influences that may also have affected the course of stress responses systems. Fourth, our sample was only composed of adult males. Because sex differences in cortisol response to stress (Carpenter et al., 2009) and sexually dimorphic associations with maltreatment have been reported (Negriff et al., 2015), although inconsistently so (Kudielka et al., 2004), our findings may not be generalizable to female participants. Fifth and finally, although HR is a fairly common measure of SNS activity, it remains highly variable and could easily be modulated by factors such as position of the participant (Vogel, Wolpert, \& Wehling, 2004), in spite of specific instructions asking 
participants to stay still at a preidentified, marked place during the TSST.

In conclusion, this study extends prior findings regarding the impact of childhood maltreatment on HPA axis and SNS reactivity to stress by reporting distinct expressions of cortisol dysregulated patterns of stress response as maltreatment increased in severity. This study calls into question the linearity assumed to exist in the association between maltreatment and

\section{References}

Allwood, M. A., Handwerger, K., Kivlighan, K. T., Granger, D. A., \& Stroud, L. R. (2011). Direct and moderating links of salivary alpha-amylase and cortisol stress-reactivity to youth behavioral and emotional adjustment. Biological Psychology, 88, 57-64. doi:10.1016/j.biopsycho.2011.06.008.

Andrews, J., Wadiwalla, M., Juster, R. P., Lord, C., Lupien, S. J., \& Pruessner, J. C. (2007). Effects of manipulating the amount of social-evaluative threat on the cortisol stress response in young healthy men. Behavioral Neuroscience, 121, 871-876. doi:10.1037/0735-7044.121.5.871.

Archer, J. (2004). Sex differences in aggression in real-world settings: A meta-analytic review. Review of General Psychology, 8, 291-322. doi:10.1037/1089-2680.8.4.291.

Bae, Y. J., Stadelmann, S., Klein, A. M., Jaeger, S., Hiemisch, A., Kiess, W., ... Dohnert, M. (2015). The hyporeactivity of salivary cortisol at stress test (TSST-C) in children with internalizing or externalizing disorders is contrastively associated with alpha-amylase. Journal of Psychiatric Research, 71, 78-88. doi:10.1016/j.jpsychires.2015.09.013.

Barnett, D., Manly, J. T., \& Cicchetti, D. (1993). Defining child maltreatment: The interface between policy and research. In T. S. Cicchetti (Ed.), Child abuse, child development, and social policy (pp. 7-73). Norwood, NJ: Ablex.

Bauer, A. M., Quas, J. A., \& Boyce, W. T. (2002). Associations between physiological reactivity and children's behavior: Advantages of a multisystem approach. Journal of Developmental and Behavioral Pediatrics, 23, 102-113. doi:10.1097/00004703-200204000-00007.

Beauchaine, T. P., \& McNulty, T. (2013). Comorbidities and continuities as ontogenic processes: Toward a developmental spectrum model of externalizing psychopathology. Development and Psychopathology, 25, 1505-1528. doi:10.1017/S0954579413000746.

Beck, A. T., Steer, R. A., \& Brown, G. K. (1996). Beck Depression Inventory-Second Edition manual. San Antonio, TX: Psychological Corporation.

Bernard, K., Frost, A., Bennett, C. B., \& Lindhiem, O. (2017). Maltreatment and diurnal cortisol regulation: A meta-analysis. Psychoneuroendocrinology, 78, 57-67. doi:10.1016/j.psyneuen.2017.01.005.

Bernstein, D., \& Fink, L. (1998). Childhood Trauma Questionnaire manual. San Antonio, TX: Psychological Corporation.

Bernstein, D., Stein, J. A., Newcomb, M. D., Walker, E., Pogge, D., Ahluvalia, T., ... Zule, W. (2003). Development and validation of a brief screening version of the Childhood Trauma Questionnaire. Child Abuse and Neglect, 27, 169-190. doi:10.1016/S0145-2134(02)00541-0.

Boks, M. P., Rutten, B. P., Geuze, E., Houtepen, L. C., Vermetten, E., Kaminsky, Z., \& Vinkers, C. H. (2016). SKA2 methylation is involved in cortisol stress reactivity and predicts the development of post-traumatic stress disorder (PTSD) after military deployment. Neuropsychopharmacology, 41, 1350-1356. doi:10.1038/npp.2015.286.

Bowes, L., \& Jaffee, S. R. (2013). Biology, genes, and resilience: Toward a multidisciplinary approach. Trauma Violence Abuse, 14, 195-208. doi: $10.1177 / 1524838013487807$.

Boyce, T. W., \& Ellis, B. J. (2005). Biological sensitivity to context: I. An evolutionary-developmental theory of the origins and functions of stress reactivity. Development and Psychopathology, 17, 271-301. doi:10.10170S0954579405050145.

Bruce, J., Fisher, P. A., Pears, K. C., \& Levine, S. (2009). Morning cortisol levels in preschool-aged foster children: Differential effects of maltreatment type. Developmental Psychobiology, 51, 14-23. doi:10.1002/dev.20333.

Bugental, D. B., Martorell, G. A., \& Barraza, V. (2003). The hormonal costs of subtle forms of infant maltreatment. Hormones and Behavior, 43, 237244. doi:10.1016/S0018-506X(02)00008-9. the stress response systems, as well as in regard to its impact on health and behavioral functioning.

\section{Supplementary Material}

To view the supplementary material for this article, please visit https://doi.org/10.1017/S0954579418000123.

Carpenter, L. L., Carvalho, J. P., Tyrka, A. R., Wier, L. M., Mello, A. F., Mello, M. F., . . Price, L. H. (2007). Decreased adrenocorticotropic hormone and cortisol responses to stress in healthy adults reporting significant childhood maltreatment. Biological Psychiatry, 62, 1080-1087. doi:10.1016/j.biopsych.2007.05.002.

Carpenter, L. L., Shattuck, T. T., Tyrka, A. R., Geracioti, T. D., \& Price, L. H. (2011). Effect of childhood physical abuse on cortisol stress response. Psychopharmacology, 214, 367-375. doi:10.1007/s00213010-2007-4.

Carpenter, L. L., Tyrka, A. R., Ross, N. S., Khoury, L., Anderson, G. M., \& Price, L. H. (2009). Effect of childhood emotional abuse and age on cortisol responsivity in adulthood. Biological Psychiatry, 66, 69-75. doi:10.1016/j.biopsych.2009.02.030.

Cicchetti, D. (2016). Socioemotional, personality, and biological development: Illustrations from a multilevel developmental psychopathology perspective on child maltreatment. Annual Review of Psychology, 67, 187-211. doi:10.1146/annurev-psych-122414-033259.

Cicchetti, D., Rogosch, F. A., Gunnar, M. R., \& Toth, S. L. (2010). The differential impacts of early physical and sexual abuse and internalizing problems on daytime cortisol rhythm in school-aged children. Child Development, 81, 252-269. doi:10.1111/j.1467-8624.2009.01393.x.

Cook, E. C., Chaplin, T. M., Sinha, R., Tebes, J. K., \& Mayes, L. C. (2012). The stress response and adolescents' adjustment: The impact of child maltreatment. Journal of Youth and Adolescence, 41, 1067-1077. doi:10.1007/s10964-012-9746-y.

Crofton, E. J., Zhang, Y., \& Green, T. A. (2015). Inoculation stress hypothesis of environmental enrichment. Neuroscience and Biobehavioral Re views, 49, 19-31. doi:10.1016/j.neubiorev.2014.11.017.

De Bellis, M. D., Lefter, L., Trickett, P. K., \& Putnam., F. W., Jr. (1994). Urinary catecholamine excretion in sexually abused girls. Journal of the American Academy of Child \& Adolescent Psychiatry, 33, 320-327. doi:10.1097/00004583-199403000-00004

de Kloet, E. R. (2014). From receptor balance to rational glucocorticoid therapy. Endocrinology, 155, 2754-2769. doi:10.1210/en.2014-1048.

Del Giudice, M., Ellis, B. J., \& Shirtcliff, E. A. (2011). The adaptive calibration model of stress responsivity. Neuroscience and Biobehavioral Re view, 35, 1562-1592. doi:10.1016/j.neubiorev.2010.11.007.

Doom, J. R., Cicchetti, D., \& Rogosch, F. A. (2014). Longitudinal patterns of cortisol regulation differ in maltreated and nonmaltreated children. Journal of the American Academy of Child \& Adolescent Psychiatry, 53, 1206-1215. doi:10.1016/j.jaac.2014.08.006

Doom, J. R., \& Gunnar, M. R. (2013). Stress physiology and developmental psychopathology: Past, present, and future. Development and Psychopathology, 25, 1359-1373. doi:10.1017/S0954579413000667.

Ellis, B. J., \& Boyce, T. (2008). Biological sensitivity to context. Current Directions in Psychological Science, 17, 183-187. doi:10.1111/j.14678721.2008.00571.x.

El-Sheikh, M., Erath, S. A., Buckhalt, J. A., Granger, D. A., \& Mize, J. (2008). Cortisol and children's adjustment: The moderating role of sympathetic nervous system activity. Journal of Abnormal Child Psychology, 36, 601-611. doi:10.1007/s10802-007-9204-6.

Elzinga, B. M., Roelofs, K., Tollenaar, M. S., Bakvis, P., van Pelt, J., \& Spinhoven, P. (2008). Diminished cortisol responses to psychosocial stress associated with lifetime adverse events: A study among healthy young subjects. Psychoneuroendocrinology, 33, 227-237. doi:10.1016/j.psyneuen.2007.11.004.

Engert, V., Efanov, S. I., Dedovic, K., Dagher, A., \& Pruessner, J. C. (2011) Increased cortisol awakening response and afternoon/evening cortisol output in healthy young adults with low early life parental care. Psychopharmacology, 214, 261-268. doi:10.1007/s00213-010-1918-4. 
Epstein, M. A., \& Bottoms, B. L. (2002). Explaining the forgetting and recovery of abuse and trauma memories: Possible mechanisms. Child Maltreatment, 7, 210-225. doi:10.1177/1077559502007003004.

Fergusson, D. M., Horwood, L. J., \& Boden, J. M. (2011). Structural equation modeling of repeated retrospective reports of childhood maltreatment. International Journal of Methods in Psychiatric Research, 20, 93-104. doi:10.1002/mpr.337.

Ferrara, P., Guadagno, C., Sbordone, A., Amato, M., Spina, G., Perrone, G., . . Corsello, G. (2016). Child abuse and neglect: A review of the literature. Current Pediatric Review. Advance online publication. doi:10.2174/ 1573396312666160914193357.

Fries, A. B., Shirtcliff, E. A., \& Pollak, S. D. (2008). Neuroendocrine dysregulation following early social deprivation in children. Developmental Psychobiology, 50, 588-599. doi:10.1002/dev.20319.

Gordis, E. B., Feres, N., Olezeski, C. L., Rabkin, A. N., \& Trickett, P. K. (2010). Skin conductance reactivity and respiratory sinus arrhythmia among maltreated and comparison youth: Relations with aggressive behavior. Journal of Pediatric Psychology, 35, 547-558. doi:10.1093/jpepsy/jsp113.

Gordis, E. B., Granger, D. A., Susman, E. J., \& Trickett, P. K. (2006). Asymmetry between salivary cortisol and alpha-amylase reactivity to stress: Relation to aggressive behavior in adolescents. Psychoneuroendocrinology, 31, 976-987. doi:10.1016/j.psyneuen.2006.05.010.

Gordis, E. B., Granger, D. A., Susman, E. J., \& Trickett, P. K. (2008). Salivary alpha amylase-cortisol asymmetry in maltreated youth. Hormones and Behavior, 53, 96-103. doi:10.1016/j.yhbeh.2007.09.002.

Grimm, K. J., Ram, N., \& Hamagami, F. (2011). Nonlinear growth curves in developmental research. Child Development, 85, 1357-1371. doi:10.1111/j.1467-8624.2011.011630.x.

Gunnar, M., \& Vazquez, D. M. (2006). Stress neurobiology and developmental psychopathology. In D. Cicchetti \& J. C. Donald (Eds.), Developmental psychopathology (Vol. 2, pp. 533-577). Hoboken, NJ: Wiley.

Gustafsson, P. E., Anckarsater, H., Lichtenstein, P., Nelson, N., \& Gustafsson, P. A. (2010). Does quantity have a quality all its own? Cumulative adversity and up- and down-regulation of circadian salivary cortisol levels in healthy children. Psychoneuroendocrinology, 35, 1410-1415. doi:10.1016/j.psyneuen.2010.04.004.

Hagan, M. J., Roubinov, D. S., Mistler, A. K., \& Luecken, L. J. (2014). Mental health outcomes in emerging adults exposed to childhood maltreatment: The moderating role of stress reactivity. Child Maltreatment, 19, 156-167. doi:10.1177/1077559514539753.

Hamstra, D. A., de Kloet, E. R., van Hemert, A. M., de Rijk, R. H., \& Van der Does, A. J. (2015). Mineralocorticoid receptor haplotype, oral contraceptives and emotional information processing. Neuroscience, 286, 412422. doi:10.1016/j.neuroscience.2014.12.004.

Harkness, K. L., Stewart, J. G., \& Wynne-Edwards, K. E. (2011). Cortisol reactivity to social stress in adolescents: Role of depression severity and child maltreatment. Psychoneuroendocrinology, 36, 173-181. doi:10.1016/j.psyneuen.2010.07.006.

Hayes, A. F. (2013). Introduction to mediation, moderation, and conditional process analysis: A regression-based approach. New York: Guildford Press.

Heim, C., Newport, D. J., Heit, S., Graham, Y. P., Wilcox, M., Bonsall, R., ... Nemeroff, C. B. (2000). Pituitary-adrenal and autonomic responses to stress in women after sexual and physical abuse in childhood. Journal of the American Medical Association, 284, 592-597. doi:10.1001/ jama.284.5.592.

Herrenkohl, T. I., Hong, S., Klika, J. B., Herrenkohl, R. C., \& Russo, M. J. (2013). Developmental impacts of child abuse and neglect related to adult mental health, substance use, and physical health. Journal of Family Violence, 28. doi:10.1007/s10896-012-9474-9.

Houtepen, L. C., Vinkers, C. H., Carrillo-Roa, T., Hiemstra, M., van Lier, P. A., Meeus, W., . . . Boks, M. P. (2016). Genome-wide DNA methylation levels and altered cortisol stress reactivity following childhood trauma in humans. Nature Communications, 7, 10967. doi:10.1038/ncomms10967.

Hunt, T. K., Slack, K. S., \& Berger, L. M. (2016). Adverse childhood experiences and behavioral problems in middle childhood. Child Abuse and Neglect, 67, 391-402. doi:10.1016/j.chiabu.2016.11.005.

Jackson, Y., Gabrielli, J., Fleming, K., Tunno, A. M., \& Makanui, P. K. (2014). Untangling the relative contribution of maltreatment severity and frequency to type of behavioral outcome in foster youth. Child Abuse and Neglect, 38, 1147-1159. doi:10.1016/j.chiabu.2014.01.008.

Juster, R. P., McEwen, B. S., \& Lupien, S. J. (2010). Allostatic load biomarkers of chronic stress and impact on health and cognition. Neuroscience and Biobehavioral Reviews, 35, 2-16. doi:10.1016/j.neubiorev.2009. 10.002 .

Kudielka, B. M., Buske-Kirschbaum, A., Hellhammer, D. H., \& Kirschbaum, C. (2004). HPA axis responses to laboratory psychosocial stress in healthy elderly adults, younger adults, and children: Impact of age and gender. Psychoneuroendocrinology, 29, 83-98. doi:10.1016/ S0306-4530(02)00146-4.

Kudielka, B. M., Hellhammer, D. H., \& Wust, S. (2009). Why do we respond so differently? Reviewing determinants of human salivary cortisol responses to challenge. Psychoneuroendocrinology, 34, 2-18. doi:10.1016/S0306-4530(02)00146-4.

Lovallo, W. R., Farag, N. H., Sorocco, K. H., Cohoon, A. J., \& Vincent, A. S. (2012). Lifetime adversity leads to blunted stress axis reactivity: Studies from the Oklahoma Family Health Patterns Project. Biological Psychiatry, 71, 344-349. doi:10.1016/j.biopsych.2011.10.018.

Lupien, S. J., McEwen, B. S., Gunnar, M. R., \& Heim, C. (2009). Effects of stress throughout the lifespan on the brain, behaviour and cognition. $\mathrm{Na}$ ture Review Neuroscience, 10, 434-445. doi:10.1038/nrn2639.

Lupien, S. J., Ouellet-Morin, I., Hupbacha, A., Tu, M. T., Buss, C., Walker, D., . . . McEwen, B. S. (2006). Beyond the stress concept: Allostatic load-A developmental biological and cognitive perspective. In D. Cicchetti \& J. C. Donald (Eds.), Developmental psychopathology (2nd ed., Vol. 2, pp. 578-628). Hoboken, NJ: Wiley.

Luthar, S. S., \& Zelazo, L. B. (2003). Research on resilience: An integrative Review. In S. S. Luthar (Ed.), Resilience and vulnerability: Adaptation in the context of childhood adversities (pp. 514-549). Cambridge: Cambridge University Press.

MacMillan, H. L., Georgiades, K., Duku, E. K., Shea, A., Steiner, M., Niec, A., . . . Schmidt, L. A. (2009). Cortisol response to stress in female youths exposed to childhood maltreatment: Results of the Youth Mood Project. Biological Psychiatry, 66, 62-68. doi:10.1016/j.biopsych.2008.

Mattei, T. A. (2014). Unveiling complexity: Non-linear and fractal analysis in neuroscience and cognitive psychology. Frontiers in Computational Neuroscience, 8, 17. doi:10.3389/fncom.2014.00017.

McEwen, B. S. (1998). Protective and damaging effects of stress mediators New England Journal of Medicine, 338, 171-179. doi:10.1056/ NEJM199801153380307.

Mead, H. K., Beauchaine, T. P., \& Shannon, K. E. (2010). Neurobiological adaptations to violence across development. Development and Psychopathology, 22, 1-22. doi:10.1017/S0954579409990228.

Meaney, M. J. (2010). Epigenetics and the biological definition of Gene $\times$ Environment interactions. Child Development, 81, 41-79. doi:10.1111/ j.1467-8624.2009.01381.x.

Mennen, F. E., Kim, K., Sang, J., \& Trickett, P. K. (2010). Child neglect: Definition and identification of youth's experiences in official reports of maltreatment. Child Abuse and Neglect, 34, 647-658. doi:10.1016/ j.chiabu.2010.02.007.

Mersky, J. P., \& Topitzes, J. (2010). Comparing early adult outcomes of maltreated and non-maltreated children: A prospective longitudinal investigation. Children and Youth Services Review, 32, 1086-1096. doi:10.1016/j.childyouth.2009.10.018.

Miller, G. E., Chen, E., \& Zhou, E. S. (2007). If it goes up, must it come down? Chronic stress and the hypothalamic-pituitary-adrenocortical axis in humans. Psychological Bulletin, 133, 25-45. doi:10.1037/00332909.133.1.25

Muthén, L. K., \& Muthén, B. O. (1998-2011). Mplus user's guide (6th ed.). Los Angeles: Author.

Negriff, S., Saxbe, D. E., \& Trickett, P. K. (2015). Childhood maltreatment, pubertal development, HPA axis functioning, and psychosocial outcomes: An integrative biopsychosocial model. Developmental Psychobiology, 57, 984-993. doi:10.1002/dev.21340.

Obradovic, J. (2012). How can the study of physiological reactivity contribute to our understanding of adversity and resilience processes in development? Development and Psychopathology, 24, 371-387. doi:10.1017/ S0954579412000053.

Ouellet-Morin, I., Odgers, C., Danese, A., Bowes, L., Shakoor, S., Papadopoulos, A., . . . Arseneault, L. (2011). Blunted cortisol responses to stress signal social and behavioral problems among maltreated/bullied 12 yearold children. Biological Psychiatry, 70, 1016-1023. doi:10.1016/j.biopsych.2011.06.017.

Ouellet-Morin, I., Wong, C. C., Danese, A., Pariante, C. M., Papadopoulos, A. S., Mill, J., \& Arseneault, L. (2013). Increased serotonin transporter gene (SERT) DNA methylation is associated with bullying victimization and blunted cortisol response to stress in childhood: A longitudinal study 
of discordant monozygotic twins. Psychological Medicine, 43, 18131823. doi:10.1017/S0033291712002784.

Parker, K. J., Buckmaster, C. L., Schatzberg, A. F., \& Lyons, D. M. (2004). Prospective investigation of stress inoculation in young monkeys. Archive of General Psychiatry, 61, 933-941. doi:10.1001/archpsyc.61.9.933.

Peckins, M. K., Dockray, S., Eckenrode, J. L., Heaton, J., \& Susman, E. J. (2012). The longitudinal impact of exposure to violence on cortisol reactivity in adolescents. Journal of Adolescent Health, 51, 366-372. doi:10.1016/j.jadohealth.2012.01.005.

Peckins, M. K., Susman, E. J., Negriff, S., Noll, J., \& Trickett, P. K. (2015). Cortisol profiles: A test for adaptive calibration of the stress response system in maltreated and nonmaltreated youth. Develpment and Psychopathology, 27, 1461-1470. doi:10.1017/S0954579415000875.

Power, C., Thomas, C., Li, L., \& Hertzman, C. (2012). Childhood psychosocial adversity and adult cortisol patterns. British Journal of Psychiatry, 201, 199-206. doi:10.1192/bjp.bp.111.096032.

Sapolsky, R. M., Romero, L. M., \& Munck, A. U. (2000). How do glucocorticoids influence stress responses? Integrating permissive, suppressive, stimulatory, and preparative actions. Endocrine Review, 21, 5-89. doi:10.1210/edrv.21.1.0389.

Saridjan, N. S., Huizink, A. C., Koetsier, J. A., Jaddoe, V. W., Mackenbach, J. P., Hofman, A., . . Tiemeier, H. (2010). Do social disadvantage and early family adversity affect the diurnal cortisol rhythm in infants? The Generation R Study. Hormones and Behavior, 57, 247-254. doi:10.1016/j.yhbeh.2009.12.001.

Shirtcliff, E. A., Peres, J. C., Dismukes, A. R., Lee, Y., \& Phan, J. M. (2014). Hormones: commentary. Riding the physiological roller coaster: Adaptive significance of cortisol stress reactivity to social contexts. Journal of Personality Disorders, 28, 40-51. doi:10.1521/pedi.2014.28.1.40.

Smith, C. A., Ireland, T. O., \& Thornberry, T. P. (2005). Adolescent maltreatment and its impact on young adult antisocial behavior. Child Abuse and Neglect, 29, 1099-1119. doi:10.1016/j.chiabu.2005.02.011.

Struber, N., Struber, D., \& Roth, G. (2014). Impact of early adversity on glucocorticoid regulation and later mental disorders. Neuroscience and Biobehavioral Reviews, 38, 17-37. doi:10.1016/j.neubiorev.2013.10.015.

Sullivan, M. W., Bennett, D. S., \& Lewis, M. (2013). Individual differences in the cortisol responses of neglected and comparison children. Child Maltreatment, 18, 8-16. doi:10.1177/1077559512449378.

Susman, E. J. (2006). Psychobiology of persistent antisocial behavior: Stress, early vulnerabilities and the attenuation hypothesis. Neuroscience and Biobehavioral Reviews, 30, 376-389. doi:10.1016/j.neubiorev.2005.08.002.
Suzuki, A., Poon, L., Papadopoulos, A. S., Kumari, V., \& Cleare, A. J. (2014). Long term effects of childhood trauma on cortisol stress reactivity in adulthood and relationship to the occurrence of depression. Psychoneuroendocrinology, 50, 289-299. doi:10.1016/j.psyneuen.2014. 09.007.

Toth, S. L., \& Cicchetti, D. (2013). A developmental psychopathology perspective on child maltreatment. Introduction. Child Maltreatment, 18 , 135-139. doi: $10.1177 / 1077559513500380$.

Trickett, P. K., Gordis, E., Peckins, M. K., \& Susman, E. J. (2014). Stress reactivity in maltreated and comparison male and female young adolescents. Child Maltreatment, 19, 27-37. doi:10.1177/1077559513520466.

Trickett, P. K., Noll, J. G., \& Putnam, F. W. (2011). The impact of sexual abuse on female development: Lessons from a multigenerational, longitudinal research study. Development and Psychopathology, 23, 453-476. doi:10.1017/S0954579411000174.

Trickett, P. K., Noll, J. G., Susman, E. J., Shenk, C. E., \& Putnam, F. W. (2010). Attenuation of cortisol across development for victims of sexual abuse. Development and Psychopathology, 22, 165-175. doi:10.1017/ S0954579409990332.

van der Vegt, E. J., van der Ende, J., Kirschbaum, C., Verhulst, F. C., \& Tiemeier, H. (2009). Early neglect and abuse predict diurnal cortisol pattern in adults: A study of international adoptees. Psychoneuroendocrinology, 34, 660-669. doi:10.1016/j.psyneuen.2008.11.004.

van Goozen, S. H., Fairchild, G., Snoek, H., \& Harold, G. T. (2007). The evidence for a neurobiological model of childhood antisocial behavior. Psychological Bulletin, 133, 149-182. doi:10.1037/0033-2909.133.1.149.

van Goozen, S. H., Matthys, W., Cohen-Kettenis, P. T., Gispen-de Wied, C., Wiegant, V. M., \& van Engeland, H. (1998). Salivary cortisol and cardiovascular activity during stress in oppositional-defiant disorder boys and normal controls. Biological Psychiatry, 43, 531-539. doi:10.1016/ S0006-3223(97)00253-9.

Voellmin, A., Winzeler, K., Hug, E., Wilhelm, F. H., Schaefer, V., Gaab, J., . . . Bader, K. (2015). Blunted endocrine and cardiovascular reactivity in young healthy women reporting a history of childhood adversity. Psychoneuroendocrinology, 51, 58-67. doi:10.1016/j.psyneuen.2014.09.008.

Vogel, C. U., Wolpert, C., \& Wehling, M. (2004). How to measure heart rate? European Journal of Clinical Pharmacology, 60, 461-466. doi:10.1007/ s00228-004-0795-3.

Young, H., \& Benton, D. (2015). We should be using nonlinear indices when relating heart-rate dynamics to cognition and mood. Scientific Reports, 5 , 16619. doi:10.1038/srep16619. 